http://jmscr.igmpublication.org/home/ ISSN (e)-2347-176x ISSN (p) 2455-0450 crossref DOI: https://dx.doi.org/10.18535/jmscr/v8i6.93

\title{
Excessive juice drinking causing symptomatic hyponatraemia in a patient with gastrointestinal pseudo - obstruction and congenital short bowel
}

\author{
Authors \\ Dhanasekhar Kesavelu', Kathleen Brown', Colin Baillie ${ }^{2}$, Wael El-Matary, \\ A Mark Dalzell ${ }^{1}$
}

${ }^{1}$ Department of Paediatric Gastroenterology, Alder Hey Children's NHS Foundation Trust, Liverpool L12 2AP, United Kingdom Liverpool L12 2AP

${ }^{2}$ Department of Paediatric Surgery, Alder Hey Children's NHS Foundation Trust, Liverpool. L12 2AP

Corresponding Author

Dr Dhanasekhar Kesavelu

Department of Paediatric Gastroenterology, Apollo Childrens hospital, Chennai, India

\section{Introduction}

Serological hyponatraemia has a number of different aetiologies. Although laboratory values may vary, the normal range for serum sodium is between $133-147 \mathrm{mmol} / \mathrm{L}$. The aetiology of hyponatraemia can be sub-divided into 3 broad categories- hypervolaemic hyponatraemia, normovolaemic hyponatraemia or hypovolaemic hyponatraemia ${ }^{(1)}$. Serum sodium levels may also appear falsely low if the blood sample is lipaemic or has high protein content.

In addition to conditions associated with antidiuretic hormone (ADH) disturbance, hyponatraemia can be caused by malnutrition in both children and adults and may become a chronic state for which replacement therapy has to be administered judiciously in order to avoid cerebral oedema.

\section{Case}

A 3-year-old boy attended an Accident and Emergency department (A\&E) with a generalised tonic clonic seizure and no previous or family history of seizure disorder. An initial serum sodium measurement was noted to be $120 \mathrm{mmol} / \mathrm{L}$, and subsequently dropped to $116 \mathrm{mmol} / \mathrm{L}$. The child was treated with intravenous hypertonic saline solution. There were no other biochemical abnormalities detected other than a urine sodium concentration measured as $9 \mathrm{mmol} / \mathrm{L}$ and a serum) potassium level of $2 \mathrm{mmol} / \mathrm{L}$. No other obvious cause for the seizure was identified. The child was prescribed $20 \mathrm{mmol}$ of oral sodium chloride 4 times daily as oral sodium supplementation, but re-presented 2 months later with a recurrence of generalised tonic clonic seizures, and a serum sodium of $113 \mathrm{mmol} / \mathrm{L}$.

The patient had a history of congenital short bowel syndrome and gastrointestinal pseudoobstruction diagnosed shortly after birth. Antenatal scanning identified an echogenic bowel at 20 weeks gestation. There was immaturity of early feeding and persistent bilious vomiting which required nasogastric and subsequent 
gastrostomy delivered feeds in order to achieve weight gain. Gastrointestinal malrotation was excluded radiologically and there was a normal sweat test and rectal biopsy to exclude Hirshsprung's disease. Due to persistent symptoms suggesting dysmotility a laparotomy was performed and the findings were of a congenital short bowel of $75 \mathrm{~cm}$ and coexistent intestinal pseudo-obstruction. The length of normal neonatal small bowel is $250 \mathrm{~cm}$ lengthening to $750 \mathrm{~cm}$ in adulthood ${ }^{(4) .}$

The child was discharged home at 5 months of age on a combination of home Parenteral Nutrition (HPN) and enteral feeds. The clinical course was complicated by central venous line sepsis and persistent feeding difficulties and feeding aversion. Enteral feeds were fully tolerated by 12 months of age and HPN was discontinued. A water deprivation test was conducted and was normal.

A detailed dietetic history at the time of admission with hyponatraemia indicated that he was consuming around 4 litres of fruit juice drink per day, with little solid food. It was concluded that this had led to pooling of fluid in dysmotile short bowel, with a subsequent fluid shift and electrolyte imbalance leading to seizure activity.

Dietary and nutritional rehabilitation was provided and up continued following discharge. There were no further episodes of hyponatremic seizures 4 years subsequently.

\section{Discussion}

Sodium levels may also fall in conjunction with a state of hyperglycaemia. The mechanism is through an increase in osmolality, and subsequent transfer of water into the extracellular space (in turn diluting the existing sodium concentration). The relationship has been described as - "Serum $\mathrm{Na}$ concentration falls about $1.6 \mathrm{mEq} / \mathrm{L}$ for every $100-\mathrm{mg} / \mathrm{dL} \quad(5.55-\mathrm{mmol} / \mathrm{L})$ rise in the serum glucose concentration above normal"(3).

A diet low in sodium logically also contributes to hyponatraemia. The table below, adapted from an online resource, indicates the sodium content of different soft drinks and juices.

Approximate $\mathrm{Na}$ Content of Common Beverages

\begin{tabular}{|l|c|}
\hline Beverage & Sodium (mEQ/L) \\
\hline Apple juice & 1.3 \\
\hline Cola & $5-6.5$ \\
\hline Diet Cola & $4.5-6.5$ \\
\hline Coffee & 1 \\
\hline Orange Juice & 3.7 \\
\hline Sports Drink & $8-33$ \\
\hline Artificial fruit drink & Trace \\
\hline
\end{tabular}

This table indicates the relatively low sodium content of soft drinks. Children relying on these fluids or drinking to excess, run the risk of inducing a hypervolaemic hyponatraemic state.

Additional gastrointestinal conditions which may potentially contribute to hyponatraemia include small bowel obstruction which may cause a 3rd space loss (which causes hyponatraemia if the replacement fluids are hypotonic).

This case report highlights the risk of fluid and electrolyte imbalance in children with underlying gastrointestinal pathology.

Congenital short bowel syndrome with pseudo obstruction is relatively rare. And infants usually present in early life with feeding problems and poor weight gain, requiring intervention with parenteral nutrition. Although parenteral nutrition is usually necessary for initial nutritional support due to a lack of adequate absorptive function, the early introduction and increase in volume of enteral feeds is crucial in order that there is adaptation of bowel function and progress to full enteral feeds ${ }^{(4-6)}$. The transition from parenteral to enteral nutrition tends to overlap with the developmental period when a weaning diet is introduced. Without adequate intestinal adaptation, there may be severe aversive feeding behaviour. This child ate very little solid food, and consumed large volumes of sugary liquid due to the lack of positive feeding experiences during the first 6 months of life.

Children's juice drinks are predominantly water and sugar, containing around $10 \%$ fruit juice by volume/concentration. The high osmotic potential 
of this fluid accounts for the dramatic electrolyte disturbance and consequent seizure activity in this child. Given the underlying diagnosis of congenital short bowel and pseudo-obstruction which led to pooling of the osmotically active drink within the short, dysmotile bowel. This case raises the importance of being aware of the risks of selective juice drinking in children with congenital gastrointestinal abnormalities.

\section{References}

1. GPnotebook.co.uk [internet]. GP Notebook - Sodium reference ranges; c2011. [Cited 2011 Feb 21]. Available from:

http://www.gpnotebook.co.uk/simplepage. cfm?ID=624230467.

2. Samadi AR, Wahed MA, Islam MR, Ahmed SM. Consequences of hyponatraemia and hypernatraemia in children with acute diarrhoea in Bangladesh. Brit Med J. 1983; 286:671-3.

3. Merck Manuals [internet]. Hyponatraemia; c2009-10. [cited 2011 Feb 22]. Available from:

http://www.merckmanuals.com/profession al/sec12/ch156/ch156d.html.

4. Cuffari C, editor. Short Bowel Syndrome [internet]. WebMD - emedicine; 2009 [cited 2011 Jan 20]. Available from: http://emedicine.medscape.com/article/931 855-overview.

5. Duro D, Kamin D, Duggan C. Overview of pediatric short bowel syndrome. J Pediatr Gastroenterol Nutr. 2008 Aug;47 Suppl 1: S33-6.

6. Hasosah M, Lemberg DA, Skarsgard E, Schreiber R. Congenital short bowel syndrome: a case report and review of the literature. Can J Gastroenterol. 2008 Jan;22(1):71-4. 